\title{
Polar coordinates in Taekwondo
}

\author{
Cristina MENESCARDI ${ }^{*}$, Isaac ESTEVAN 2 , \& Coral FALCO 3 \\ ${ }^{1}$ Catholic University "San Vicente Mártir" of Valencia (Spain) \\ 2 University of Valencia (Spain) \\ 3 University of Bergen (Norway)
}

5th IMACSSS World Scientific Congress Abstracts, Rio Maior (Portugal), October 6-8

Section: Coaching, training and health issues in MA\&CS

Type: Oral communication

\section{Introduction}

In observational methodology, inter-relational behaviors are relevant for coaches and athletes to know which behaviors are dependent or inexistent while others occur. Polar coordinates analysis allows researchers to provide an inter-relational map of behaviors (from focal to conditioned ones) represented by vectors (Anguera \& Hernandez-Mendo, 2014).

To date, there is only one study in taekwondo that applied this statistical technique (López-López, Menescardi, Estevan, Falco, \& Hernández-Mendo, 2015). This study showed a relationship of anticipatory counterattack actions with effective attacks performed by using kicks to the chest (López-López, et al., 2015). The revision of the current literature suggested a lack of behavioral patterns studies in combat sports. Thus, the purpose of this study is to analyze the relationships between tactical actions (direct attack [DA], indirect attack [IA], anticipatory counterattack [AC], simultaneous counterattack [SC], and posterior counterattack [PC]) of taekwondo to extract behavioral patterns which could be used to train the tactics based on empirical evidences.

\section{Methodology}

A Generalizability analysis was previously applied to determine the generalizabilization of the sample size to the universe (Blanco-Villaseñor, Castellano, Hernández-Mendo, Sánchez- López, \& Usabiaga, 2014). Six male combats from the 2012 Olympic Games were analyzed. HOISAN software was used to codify the behavior from the videotapes, analyze the polar coordinates and represent the respective vector in their quadrant (Hernández-Mendo, López-López, Castellano, Morales, \& Pastrana, 2012). Following procedures from previous studies (López-López, et al., 2015), significant patterns were determined with radium $>1.96$ in one lag anterior and posterior to the focal behaviour. Five polar coordinates analyses were conducted, each of them with every tactical action as a focal behaviour.

\section{Results}

Regarding attacking actions (DA and IA) as focal behavior, counterattacks (AC, SC, and PC) appeared in quadrant I, while attacking actions (DA and IA) appeared in the quadrant III. For AC as focal behavior, DA and IA appeared in quadrant I. For SC as focal behavior, attacks (DA, and IA) appeared in quadrant I while counterattacking actions (AC, SC, and PC) appeared in quadrant III. Finally, for PC as focal behavior, DA and IA appeared in quadrant I, and SC and PC in the quadrant III. 


\section{Discussion and conclusion}

Results of the current study show that when attacking actions (DA and IA) are conducted as focal behaviors, an excitatory relationship exists with counterattacking actions (AC, SC, and PC) in both, retrospective and prospective, perspectives (quadrant I), while the performance of DA and IA is inhibited (quadrant III). Also, when counterattacking actions (AC, SC and PC) are conducted as focal behaviors, attacking actions (DA, and IA) are activated in both perspectives (quadrant I) and counterattacking ones are inhibited in both perspectives (quadrant I). The lack of relationship between SC and PC with AC could be due to the scare presence of this action. In short, independently of the attacking action performed (DA or IA) whatever counterattacking action (AC, SC or PC) were related (i.e., preceded or followed) to this action. Furthermore, similar conditions existed for counterattacking actions, which are preceded and followed by either attacking action (DA or IA). These results seem congruent due to the internal logic of taekwondo wherein an attack is followed by a counterattack, and so on (Joseph, 2012). A posterior sequential analysis must be carried out so that a clearer pattern of the relationships between behaviors can be shown. These results suggest including defensive actions as blocks while DA, IA, AC and SC are performed due to the simultaneity of actions, to avoid the opponent's score in addition to train the timing in perform the PC when DA or IA is performed.

\section{References}

Anguera, M.T., \& Hernández-Mendo, A. (2014). Metodología observacional y psicología del deporte. Revista de Psicología del Deporte, 23(1), 103-109.

Blanco-Villaseñor, A., Castellano, J., Hernández-Mendo, A., Sánchez- López, C.R., \& Usabiaga, 0. (2014). Aplicación de la TG en el deporte para el estudio de la fiabilidad, validez y estimación de la muestra. Revista de Psicología del Deporte, 23(1), 131-137.

López-López, J. A., Menescardi, C., Estevan, I., Falco, C., \& Hernández-Mendo, A. (2015). Análisis técnico-táctico en Taekwondo con coordenadas polares a través del software HOISAN. Cuadernos de Psicología del Deporte, 15(1), 131-142.

Hernández-Mendo, A., López-López, J.A., Castellano, J., Morales, V., \& Pastrana, J.L. (2012). HOISAN 1.2: Programa informático para uso en metodología observacional. Cuadernos de Psicología del Deporte, 12(1), 55-78.

Joseph, J. (2012). The practice of capoeira: diasporic black culture in Canada. Ethnic and racial studies, 35(6), 1078-1095.

Key words: Taekwondo; behavior patterns; Olympic Games; combat sports.

Study supported by the project of the 'Consellería de Educación, Investigación y Deporte' ACIF/2014/268 (Generalitat Valenciana). 